\title{
Clinicopathological features and prognosis of patients with esophageal cancer as the second primary cancer: a large population-based analysis using the SEER program [2000-2015]
}

\author{
Zhencong Chen", Ming Li", Ke Ma*, Zhengyang Hu, Shuai Wang, Hongyu Chen, Yuansheng Zheng, \\ Cheng Zhan, Zongwu Lin, Qun Wang \\ Department of Thoracic Surgery, Zhongshan Hospital, Fudan University, Shanghai 200032, China \\ Contributions: (I) Conception and design: Z Chen, M Li, C Zhan, Z Lin, Q Wang; (II) Administrative support: C Zhan, Z Lin, Q Wang; (III) \\ Provision of study materials or patients: Z Chen, M Li, K Ma; (IV) Collection and assembly of data: Z Chen, M Li; (V) Data analysis and \\ interpretation: Z Chen, K Ma; (VI) Manuscript writing: All authors; (VII) Final approval of manuscript: All authors. \\ \#These authors contributed equally to this work. \\ Correspondence to: Cheng Zhan; Zongwu Lin. Department of Thoracic Surgery, Zhongshan Hospital, Fudan University, No. 180, Fenglin Road, \\ Shanghai 200032, China. Email: czhan10@fudan.edu.cn; lin.zongwu@zs-hospital.sh.cn.
}

\begin{abstract}
Background: This study used the Surveillance, Epidemiology, and End Results (SEER) data to investigate clinicopathological characteristics and prognoses of patients with esophageal cancer as a second primary cancer from 2000 to 2015.

Methods: Esophageal cancer as the second primary cancer (SEPC) patient information collected from the SEER database were extracted, evaluated, and compared with only primary esophageal cancer (OPEC) patient data. Overall survival (OS) was evaluated by the Kaplan-Meier method. Univariate analysis (UVA) and multivariate analysis (MVA) by the Cox proportional hazards regression identified risk factors that predicted OS and the results were used to construct a nomogram to predict OS in SEPC patients.

Results: A total of 7,161 esophageal cancer patients were identified from the SEER registry, which included 813 patients $(11.4 \%)$ with SEPC in the patient history. The most common cancer for the first primary cancer prior to esophageal cancer (FPC) was genital system cancer (43.5\%). The median OS was 7 (95\% CI: 6.69-11.31) months and was not significantly different from that of patients with OPEC. The patients with SEPC appeared with unique clinical pathological features compared with OPEC, including older age, more squamous cell carcinoma, and early stage esophageal cancer, and less lower third of esophageal cancer $(\mathrm{P}<0.001)$. UVA and MVA identified M stage, surgery, and chemotherapy as independent risk factors. The nomogram had a calibration index of 0.682 .
\end{abstract}

Conclusions: SEPC had unique clinicopathological characteristics. M stage, surgery, and chemotherapy were independently associated with OS. The nomogram accurately predicted OS.

Keywords: Esophageal cancer as the second primary cancer (SEPC); clinicopathological characteristics; survival; nomogram; Surveillance, Epidemiology, and End Results database (SEER database)

Submitted Sep 24, 2019. Accepted for publication Dec 12, 2019.

doi: $10.21037 /$ tcr.2019.12.64

View this article at: http://dx.doi.org/10.21037/tcr.2019.12.64

\section{Introduction}

Esophageal cancer is one of the most common causes of cancer-related death, and is the sixth leading cause of death worldwide, responsible for over 500,000 cases every year (1). There have been numerous studies reporting the clinicopathological features and prognostic factors of esophageal cancer $(2,3)$. Despite the management of esophageal cancer patients has been greatly improved, 
the general outcome remains very poor for overall 5 -year survival rates $(10 \%)$ and 5 -year post esophagectomy survival rates (15-40\%) (4). Although the development of a second primary cancer after an index esophageal cancer is fairly rare, with the rapid development of treatment technology and the population continuing to age, the number of elderly patients with esophageal cancer as a second primary cancer following other cancer history is expected to further increase in the near future (5). Under such circumstances clinicopathological characteristics and prognosis factors of second cancer should be studied in depth. With the increasing incidence of second primary cancer in subjects with esophageal cancer, there may be benefit and essential to accurately predicting the prognosis. However, almost all studies only focused on the first primary esophageal cancer. Studies of the patients with other tumor histories are rare.

As a large population-based source for cancer statistics, the Surveillance, Epidemiology, and End Results (SEER) database provides detailed clinical and survival information of patients with esophageal cancer. In this study the SEER database was used to investigate the clinicopathological characteristics and prognosis factors of second primary cancers in the esophagus, to help understand this unexplored subject.

\section{Methods}

We used the SEER database from 2000 and 2015, and patients were selected by SEER ${ }^{*}$ Stat software version 8.3.5 (https://seer.cancer.gov/seerstat/). The criteria of inclusion were as follows: (I) all cases were identified as esophageal cancer by using topography codes (C150-C155 and C158-C159) of the International Classification of Diseases for Oncology, 3rd Edition ICD-O-3 and with a site recode ICD-O-3/WHO 2008 using esophagus; (II) every patient had their own ID in the SEER database, and patient IDs were used to match patients whose esophageal cancer was the second primary tumor in the patient history (included patients), and patients whose esophageal cancer was the only primary tumor in the patient history were included as controls; (III) histologically confirmed malignant esophageal cancer, with complete follow-up data were included. The exclusion criteria were any case without histology or histology type not defined or unknown, T, N or M stages not specified, or survival information unknown.

We collected the following information for each patient from the SEER database: (I) the demographics of patients (age at diagnosis, sex, and race); (II) characteristics of tumors (tumor primary site, tumor size, pathological grade, TNM stage of the primary tumor, and histological type); therapy details (surgery, radiotherapy, and chemotherapy); and follow-up records (cause of death, cancer-specific death, and survival months). The TNM stage was manually adjusted following the AJCC eighth edition criteria (6).

\section{Ethical statement}

Ethics approval was exempted by the Ethics Committee of the Zhongshan Hospital, Fudan University (Shanghai, China), as the SEER is a publicly available database, and data extracted from SEER were identified as an exempted study.

\section{Statistics analysis}

Statistical analyses were performed using IBM SPSS statistics software, version 22.0 (IBM, Inc., Armonk, NY, USA) and R version 3.5.1 (R Foundation for Statistical Computing, Vienna, Austria). The R package included survival, rms, and ggplot2. Kaplan-Meier and log-rank tests were used to construct and compare survival curves. Patient variables with prognostic values were identified using Cox proportional hazards regression and reported as hazard ratios (HRs). Based on the multivariate regression analysis, the nomogram was integrated to further express the relationship between the significant variables in the prediction model. A two-tailed $\mathrm{P}$ value of $<0.05$ was considered statistically significant. Variables whose $\mathrm{P}$ values were $<0.1$ in univariate analyses were included in multivariate analyses.

The results of MVA were used to structure a nomogram and concordance index (c-index) and calibration plots were used to evaluate model performance as previously reported (7). The C-index ranges from 0.5 to 1.0 and a larger C-index indicated better accuracy for predicting prognoses. In addition, nomograms were used to compare the predicted survival with the observed survival.

\section{Results}

\section{Patient characteristics}

A total of 7,161 esophageal cancer patients were identified from the SEER registry, which included 813 patients (11.4\%) whose esophageal cancers were the second primary tumor in patient history, and 6,348 patients $(88.6 \%)$ whose 
esophageal cancers were the only primary tumors in patient history. The baseline demographic, clinical, and pathological characteristics of all participants are summarized in Table 1. Patients with esophageal cancer as the second primary cancer (SEPC) included 679 men and 134 women with a median tumor size of $50 \mathrm{~mm}$ and interquartile range (IQR) of 30-70 mm. SEPC patients had a median age of 73 years and the majority of patients in this dataset were Caucasian $(82.2 \%)$. The median year after primary cancer diagnosis was 7.29 and the IQR was 2-10 years. The histological grade was categorized into two groups: 44\% grades I and II (well differentiated or moderately differentiated); 56\% grades III and IV (poorly differentiated or undifferentiated). The most common AJCC stage was stage IV (29.6\%), followed by stage III (28.7\%), and stage II (22.3\%). Of these patients, only 132 patients (16.2\%) underwent surgery and as many as 506 patients had accepted radiation therapy and 507 patients $(62.4 \%)$ had undergone chemotherapy.

Compared with only primary esophageal cancer (OPEC) patients, SEPC patients had significantly more early stage tumors $(\mathrm{P}<0.001)$, older age $(\mathrm{P}<0.001)$, more squamous cell carcinoma $(\mathrm{P}<0.001)$, fewer lower third of esophageal cancer $(\mathrm{P}<0.001)$, and were less likely to have had chemotherapy $(\mathrm{P}=0.021)$.

\section{The first primary cancer prior to esophageal cancer (FPC)}

The cases of FPC are shown in Table S1. The most common cancer for FPC was genital system cancer (43.5\%), followed by respiratory system cancer $(12.3 \%)$, oral cavity and pharynx cancer (10.5\%), and urinary system cancer $(10.2 \%)$. Other SEPC patients were diagnosed with cancers derived from the colon and rectum (9.8\%), lymphoma (5.8\%), skin excluding basal and squamous (4.4\%), and other organs $(3.5 \%)$.

\section{Survival analyses}

The survival outcomes for SEPC patients and OPEC patients are shown in Figure 1. SEPC patients whose median overall survival (OS) was 7 (95\% CI: 6.69-11.31) months, 3-year OS was 3.20\% (95\% CI: 4.38-2.02\%), and 5 -year OS was only $0.60 \%$ (95\% CI: $0.01-1.19 \%)$ are shown. As shown in Figure 1, the OS was not significantly different in SEPC vs. OPEC patients.

A total of 813 patients with SEPC were included in univariate analyses and multivariate analyses to find the predictors of survival. As Figure 2 shows, age $(\mathrm{P}=0.318)$, sex $(P=0.764)$, race $(P=0.196)$, and year after primary cancer $(\mathrm{P}=0.481)$ diagnosis were not significant prognostic indicators for SEPC patients.

We further examined the correlation between survival and other parameters. As shown in Figure 3, AJCC stage $(\mathrm{P}=0.002)$ was a significant prognostic indicator for SEPC patients, while others were not. As shown in Figure 4, patients who underwent surgery $(\mathrm{P}<0.001)$, chemotherapy $(\mathrm{P}<0.001)$, or radiotherapy $(\mathrm{P}<0.001)$ had significantly better OS than those who did not, and univariate analyses revealed that $T$ stage $(\mathrm{P}<0.001)$ and $M$ stage $(\mathrm{P}<0.001)$ were risk factors, while $\mathrm{N}$ stage $(\mathrm{P}=0.701)$, tumor size $(\mathrm{P}=0.348)$, and location $(\mathrm{P}=0.984)$ did not significantly affect $\mathrm{OS}$.

\section{Cox regression analysis}

All risk factors whose $\mathrm{P}$ value was $<0.1$ in univariate analyses were applied to multivariate analyses, except stage, considering that stage was relevant to $\mathrm{T}, \mathrm{N}$, and $\mathrm{M}$. The results showed that, $M$ stage $(P=0.007)$, surgery $(P<0.001)$, and chemotherapy $(\mathrm{P}<0.001)$ remained independent prognostic predictors for SEPC patients, which could predict survival. Grade, histological type, $\mathrm{T}$ and $\mathrm{N}$ stage, radiation, and the location of FPC were not independent prognostic predictors. The details of the correlations between survival and factors mentioned above are shown in Table 2.

\section{Construction and validation of a nomogram}

A nomogram, which was based on the significant risk factors including three factors ( $M$ stage, surgery and chemotherapy) identified in multivariate analyses, is shown in Figure 5. OS could be predicted by adding the points corresponding to the patient's characteristics. The c-index of this nomogram was 0.724 (95\% CI: 0.721-0.727), which showed good predictive ability. The calibration plot based on bootstrap resampling validation is illustrated in Figure S1, which was nearly a diagonal line, indicating a good prediction model. SEPC total points of patients in the nomogram $(\mathrm{P}<0.001)$ were significantly related to OS (Figure $S 2)$.

\section{Discussion}

In this study, 800 patients with SEPC were identified in the SEER database. Compared with OPEC patients, SEPC patients had significantly more early stage tumors, older age, less adenocarcinoma, fewer lower third of 
Table 1 Baseline characteristics of SPEC and OPEC patients

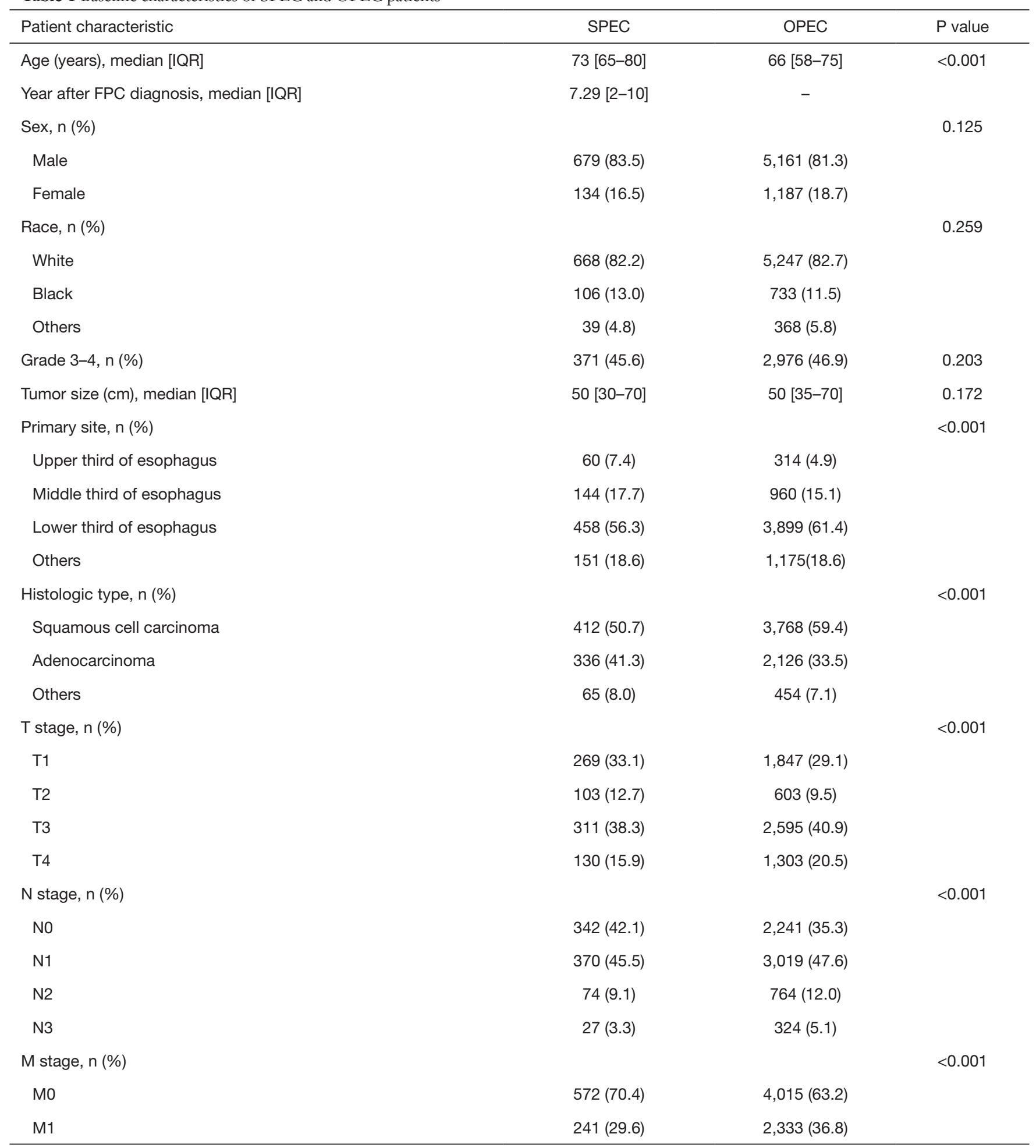

Table 1 (continued) 
Table 1 (continued)

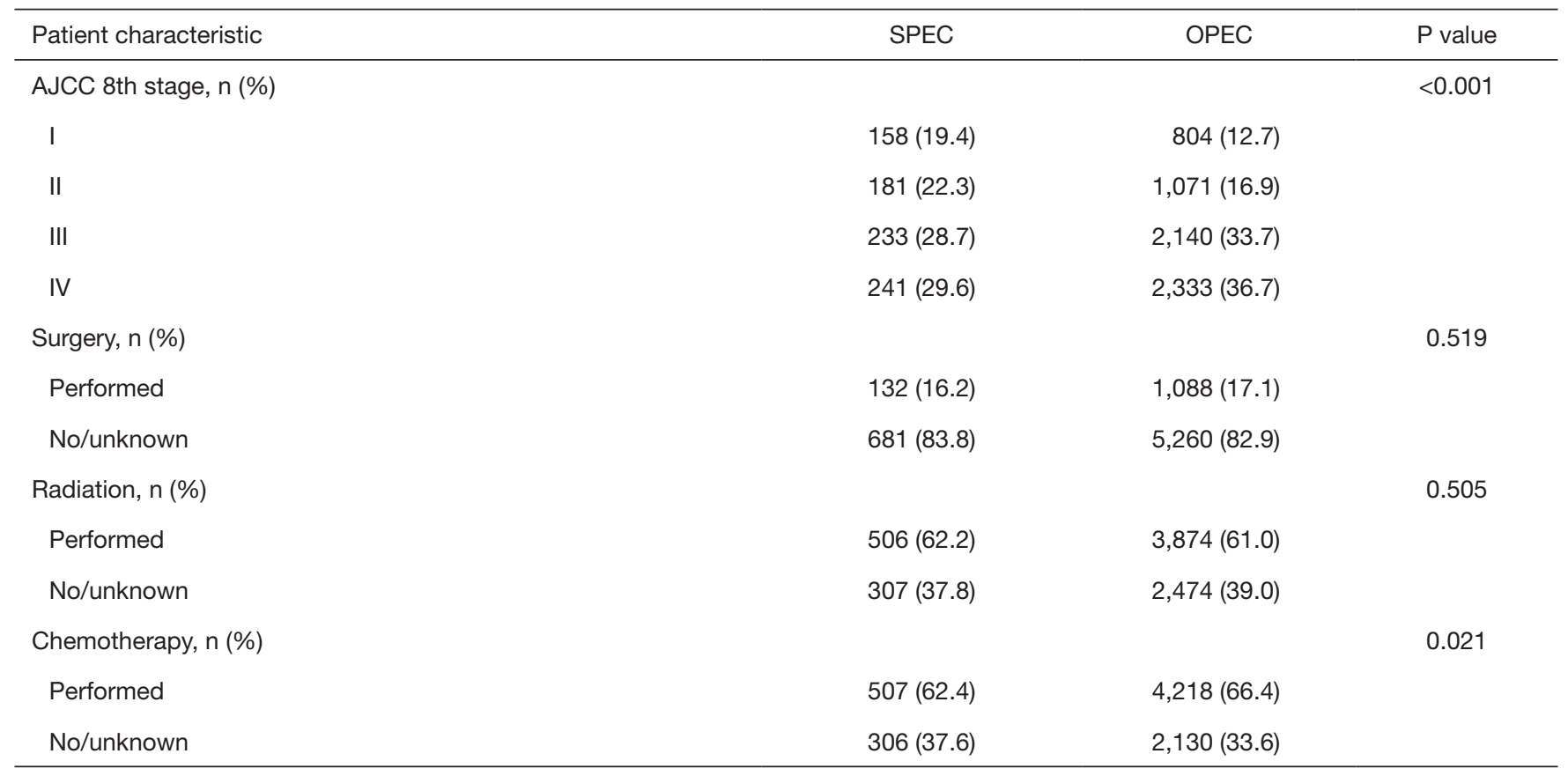

SPEC, esophageal cancer as the second primary cancer; OPEC, only primary esophageal cancer; IQR, interquartile range; OS, overall survival.

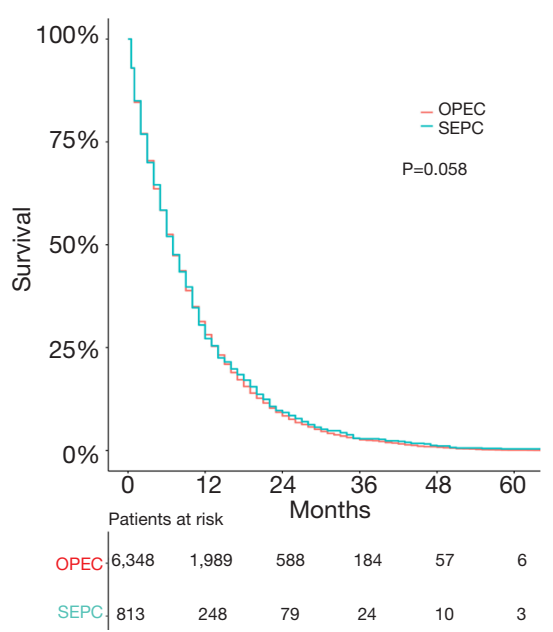

Figure 1 Kaplan-Meier estimate of overall survival of SEPC patients and OPEC patients. SEPC, second primary cancer; OPEC, only primary esophageal cancer.

esophageal cancer, and were less likely to have undergone chemotherapy. The OS of patients with SPEC and OPEC were similar. $M$ stage, surgery, and chemotherapy were found to be independently associated with survival. A nomogram based on three significant factors ( $M$ stage, surgery, and chemotherapy) was built to visually and effectively predict the 1-, 2-, and 3-year OS rates of SPEC patients.

Previous studies have suggested the most common FPC sites for SEPC are aerodigestive tract organs, such as the oral cavity, pharynx, larynx, and lung, and the three leading first primary cancer sites were oral cavity, pharynx, and respiratory system, which could be explained by a "field cancerization" effect (8-10). According to epidemiological reports that cancer of the tongue, pharynx, and esophagus are caused by the same carcinogens (such as alcohol and tobacco) and carcinogenic effects, which lead to cancers on the aerodigestive tract that can act on the esophagus at the same time, this could activate the development of esophageal cancer. The genital system, oral cavity and pharynx, and respiratory system were the top three types in our study. Muller et al. reported that the genital system can be a common site for SEPC (11). This finding may be explained by radiotherapy or chemotherapy having become a traditional measure to treat the genital system $(12,13)$, so the sequelae of treatment, especially the side effects, which appear after radiotherapy treatment, along with other adverse stimulation, may contribute to the development of SEPC $(14,15)$. Additionally, in our study, radiotherapy 

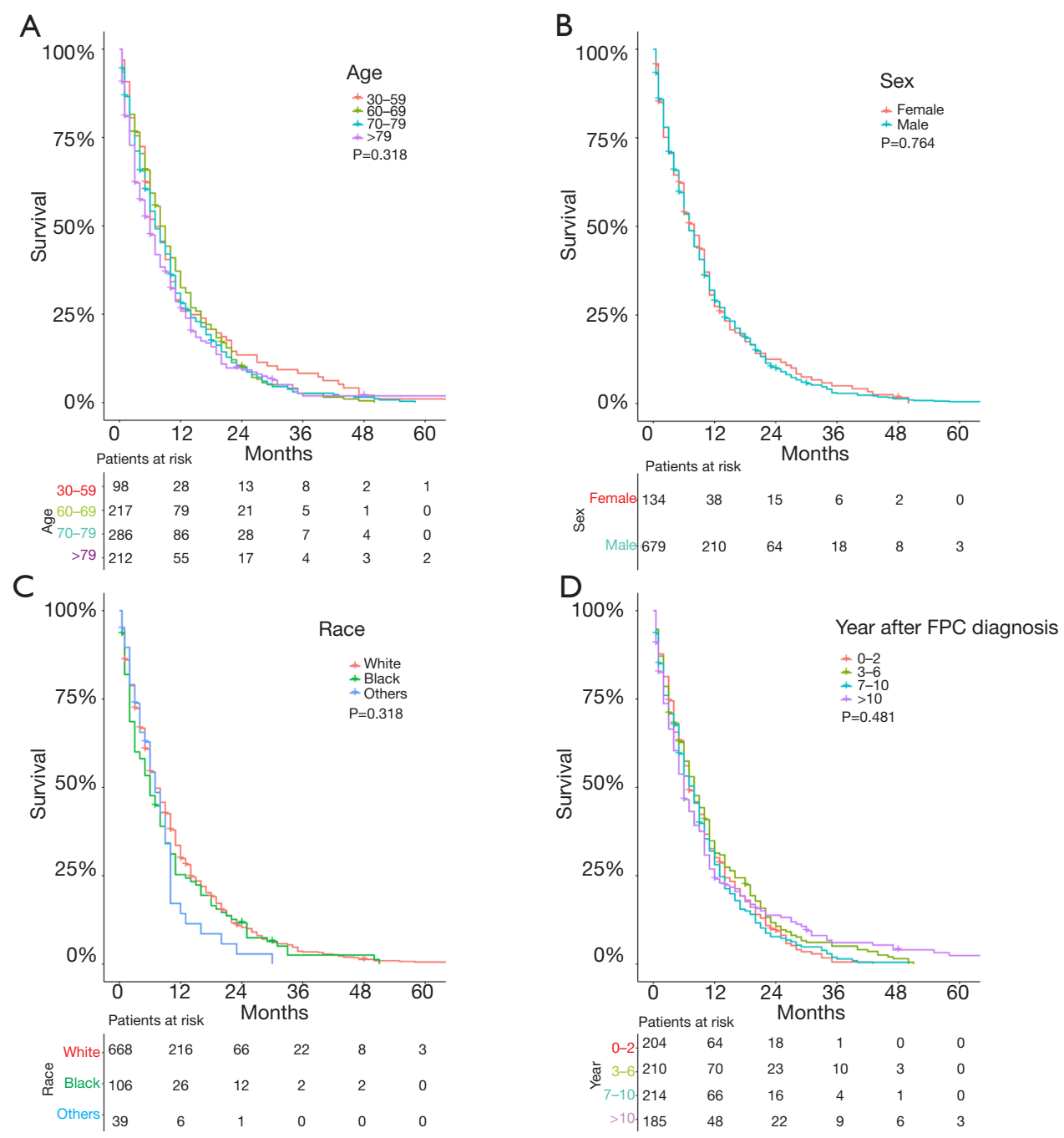

Figure 2 Kaplan-Meier estimate of overall survival of patients by (A) age, (B) sex, (C) race, and (D) year after FPC diagnosis. FPC, first primary cancer.

was not an independent risk factor, which can influence the survival of SEPC, in agreement with a past report (16). The problem of SEPC among genital system cancer survivors may be related to treatment side effects or to etiological associations for multiple cancers.

In contrast, in our study, patients who had chemotherapy had a better OS than patients without chemotherapy, which was consistent with a previous study (17). Thus, we urge further investigation to establish a balance between radiotherapy and chemotherapy treatments. Our study also demonstrated that some sites can be FPC sites, such as in the colon, rectum, and lymphoma, which was consistent with previous studies $(18,19)$, while some other cancers have lower incidences, such as for mesothelioma, or a high mortality, such as pancreatic cancer and liver cancer.

To our knowledge, SEPC patients should have a poorer prognosis than OPEC patients (20). Lee et al. reported that worse nutritional status, older age, significantly lower serum albumin, and body mass index may contribute to the poorer prognosis. Nevertheless, studies also reported that patients with OPEC did not have significantly better OS than SEPC patients $(21,22)$. Additionally, there was only one study focusing on this theme, which included more than 800 patients with esophageal cancer. Poon et al. (23) used a database of 1,055 patients in Hong Kong, and found no differences in survival between SEPC patients and OPEC patients. In our study, the OS of patients with SEPC and OPEC were similar, with a median OS of SEPC patients 


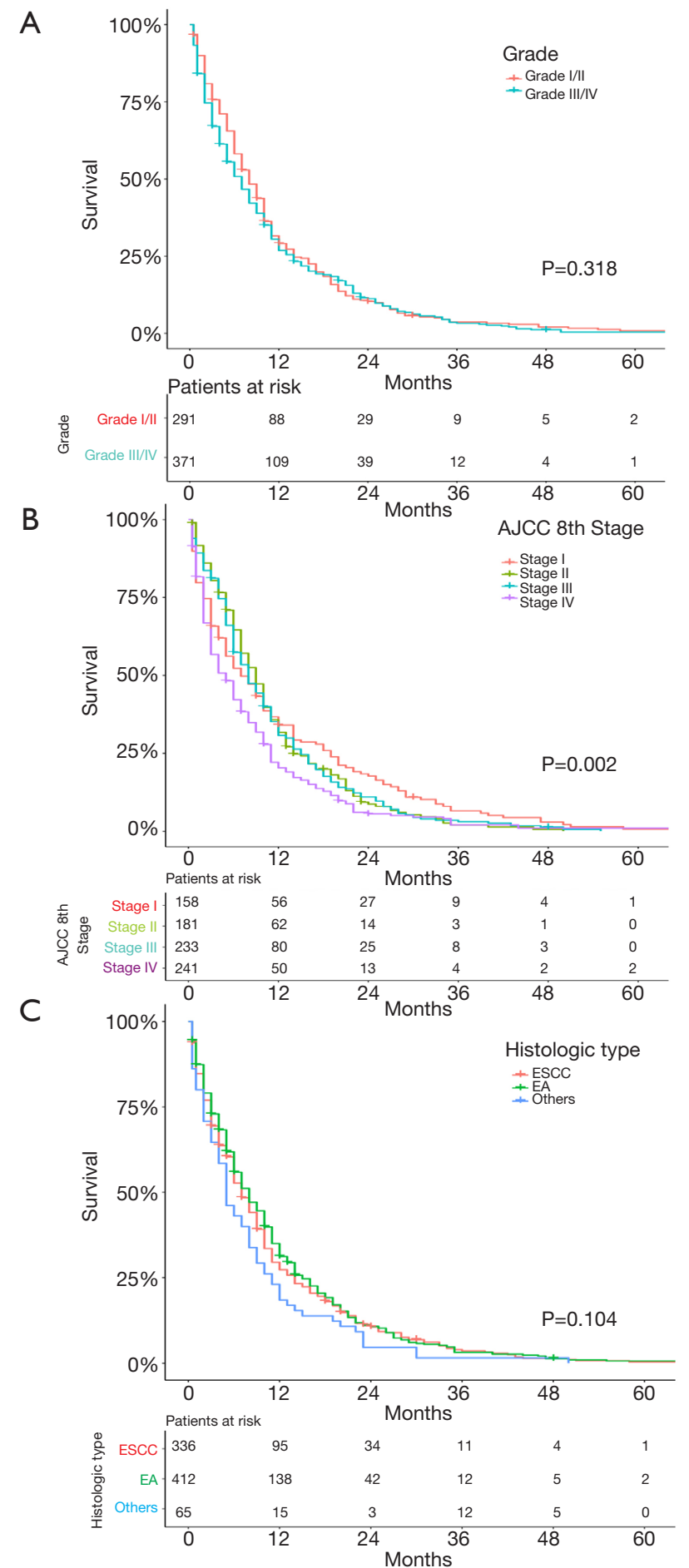

Figure 3 Kaplan-Meier estimate of overall survival of patients by (A) histological grade, (B) AJCC stage, and (C) histological type.
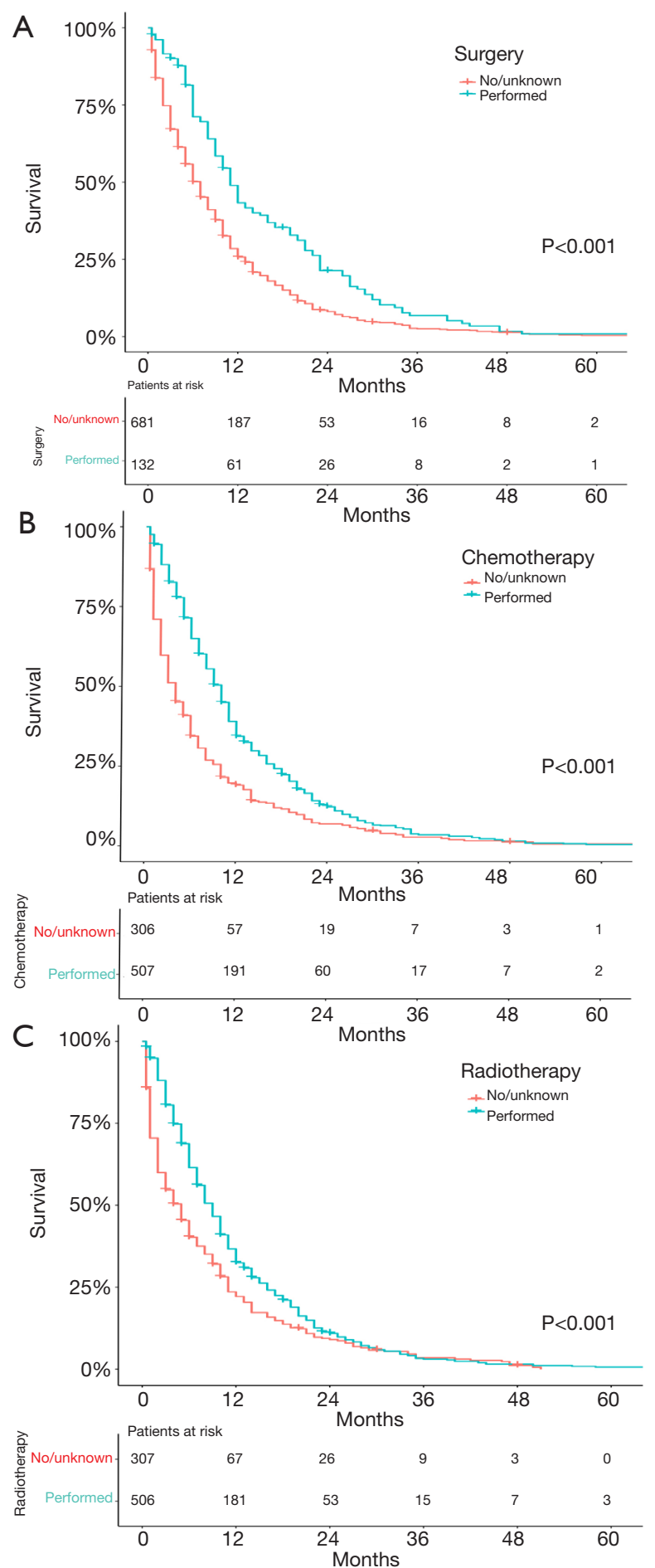

Figure 4 Kaplan-Meier estimate of overall survival in patients treated by (A) surgery, (B) chemotherapy, or (C) radiotherapy. 

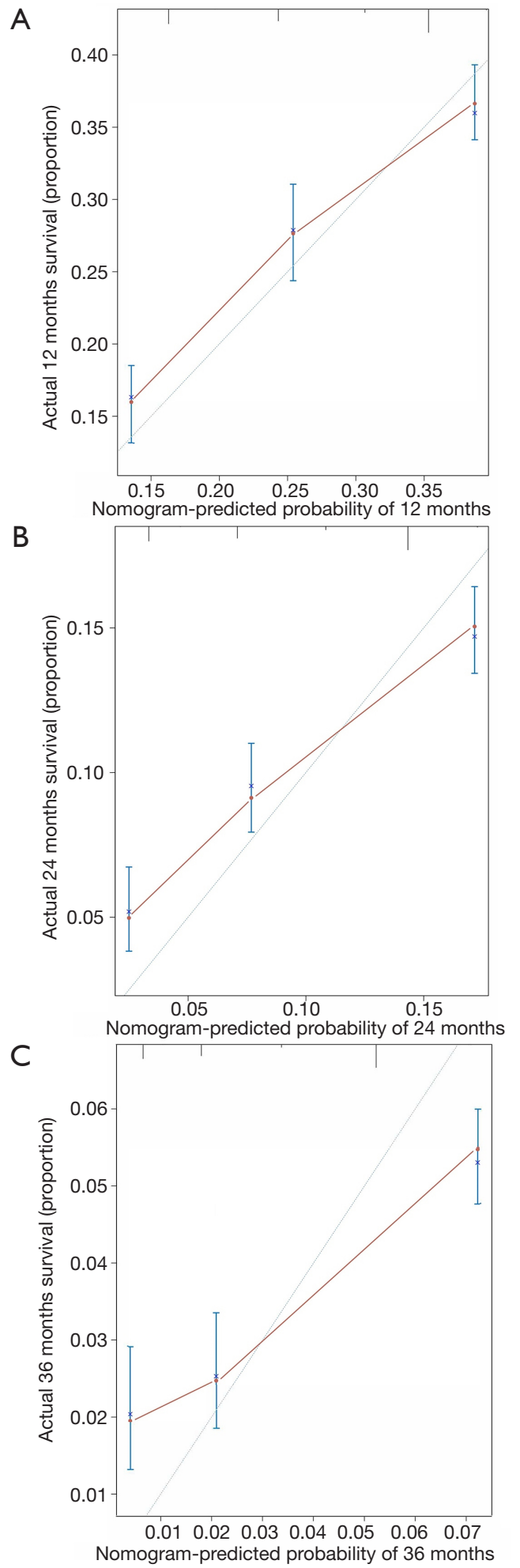

Figure 5 Calibration plots of the nomogram prediction of (A) 1-year, (B) 2-year, and (C) 3-year overall survival of SEPC patients. The red line represents equality of the observed and predicted probability. SEPC, second primary cancer. and OPEC patients both being 7 months, which agreed with previous studies. Male patients comprised a larger percentage than female patients for both SEPC and OPEC patients (males comprised over $80 \%$ in both SEPC and OPEC groups), and other studies also showed a strong male predominance in study cohorts $(20,22)$, with males more likely to have had exposures to tobacco and alcohol. This may be the reason for the male predominance.

Kumagai et al. reported that SEPC was commonly related to more I stages and less IV stages than OPEC $(24,25)$. This result was consistent with that of our study. It was suggested that because patients with FPC may have more chance to check on their health, they were more likely to have early stage EC. In our study, we found the most common location of EC was the lower third of the esophagus, and histological grades were similar between SEPC and OPEC patients, which confirmed the results of previous studies $(20,26)$. However, patients with SEPC had fewer lower third EC and had more adenocarcinoma than OPEC patients in our study. However, the results were difficult to compare because of differences in the study cohorts, methods for calculating data, or simply chance variations may have caused these differences.

$M$ stage, surgery, and chemotherapy were independent risk factors following MVA, as shown with a nomogram based on these risk factors. Intriguingly, some of the TNM descriptors like $\mathrm{T}$ and $\mathrm{N}$ were not significant in our univariate or multivariate analyses. After the deep consideration, we thought it was because the lumen structure and anatomy of the esophagus had been changed and rebuilt both in patients with surgery or non-surgery such as radiotherapy and chemotherapy. For those patients who underwent surgery with the primary esophageal cancer, their lymph nodes around the esophageal had been dissected and it was difficult or meaningless to evaluate accurately again when the patients had the secondary esophageal cancer. Besides, the sample size, or poorly description in the database may also have an effect on our results.

In our nomogram, surgery had a strong impact on OS, and patients who had surgery had a better OS. Otowa et al. reported that surgical resection could be safely performed in SEPC patients despite the other factors, such as more blood loss or longer operation time that could complicate the procedure (22). In a previous study, surgery was a preferred treatment for patients whose time of diagnosis between FPC and SEPC was more than 1 year (16), especially in FPC of neck and head cancers, and the benefits of surgery were greater with resection at 
Table 2 Univariate and multivariate Cox proportional hazards analysis

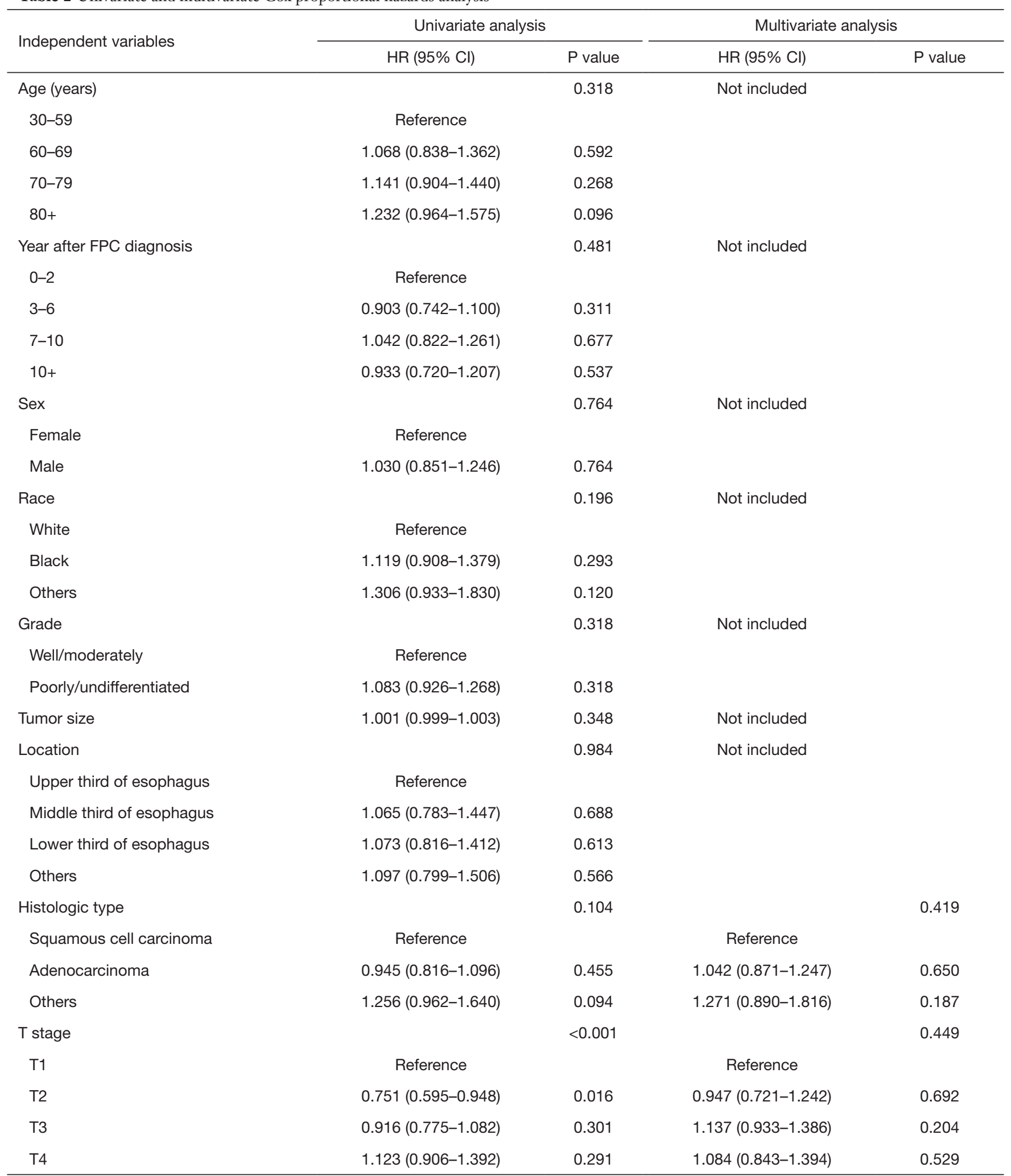

Table 2 (continued) 
Table 2 (continued)

\begin{tabular}{|c|c|c|c|c|}
\hline Independent variables & \multicolumn{2}{|c|}{ Univariate analysis } & \multicolumn{2}{|c|}{ Multivariate analysis } \\
\hline $\mathrm{N}$ stage & & 0.701 & Not included & \\
\hline No & Reference & & & \\
\hline $\mathrm{N} 1$ & $1.095(0.942-1.247)$ & 0.238 & & \\
\hline N3 & $1.080(0.729-1.599)$ & 0.703 & & \\
\hline M stage & & $<0.001$ & 0 & 0.007 \\
\hline MO & Reference & & Reference & \\
\hline M1 & $1.342(1.150-1.567)$ & $<0.001$ & 1.283 (1.072-1.535) & 0.007 \\
\hline III & $1.095(0.891-1.347)$ & 0.388 & & \\
\hline IV & $1.424(1.157-1.751)$ & 0.001 & & \\
\hline Surgery & $<0.001$ & & & $<0.001$ \\
\hline No/unknown & Reference & & Reference & \\
\hline Performed & $0.623(0.513-0.756)$ & $<0.001$ & $0.650(0.526-0.804)$ & $<0.001$ \\
\hline Radiation & & $<0.001$ & 0 & 0.063 \\
\hline No/unknown & Reference & & Reference & \\
\hline Skin* & Reference & & Reference & \\
\hline Lymphoma & 1.095 (0.704-1.703) & 0.687 & $1.088(0.678-1.746)$ & 0.725 \\
\hline Colon and rectum & $0.952(0.637-1.422)$ & 0.810 & $0.968(0.634-1.479)$ & 0.882 \\
\hline Urinary system & $1.214(0.816-1.805)$ & 0.339 & $1.307(0.855-1.998)$ & 0.216 \\
\hline Oral cavity and pharynx & $1.079(0.725-1.606)$ & 0.707 & $1.166(0.763-1.782)$ & 0.478 \\
\hline Respiratory system & $1.390(0.944-2.046)$ & 0.095 & $1.335(0.882-2.020)$ & 0.172 \\
\hline Male genital system & $1.061(0.747-1.507)$ & 0.740 & $1.097(0.761-1.582)$ & 0.620 \\
\hline Others & $1.465(0.951-2.258)$ & 0.083 & $1.457(0.906-2.343)$ & 0.120 \\
\hline
\end{tabular}

*, skin excluding basal and squamous. FPC, first primary cancer prior to esophageal cancer.

an early stage $(27,28)$. While for patients whose time of diagnosis between FPC and SEPC was less than one year, Lee showed surgical treatment also could be an established strategy (29). However, although the ideal surgery methods for SEPC included such factors as tumor location, patient conditions, the surgeon's preference, and expertise, the study still showed that postoperative mortality rates were relatively high (range, $8.5-9.3 \%)(24,26)$. Lee et al. reported 
that surgical treatment for SEPC patients may not be the optimal choice due to technical complexity, different biological behaviors of multiple tumors, extra costs, and increased patient anxiety (20). However, patients must be carefully evaluated before the decision for surgery, thus additional research is needed to decide whether to perform operations. In difficult cases, chemoradiotherapy served as an optimal alternative (30). However, additional research is needed to resolve this important question.

There were several limitations in this study. First, it was possible that the sample size may have contributed to a lack of association, such as having no cases of liver cancers as FPC from the SEER database (date not shown). Second, because the SEER database did not provide detailed information (such as the details of chemotherapy or radiotherapy), and we could not explore those variables since the incomplete data in our study. Third, the patients were from the USA, thus, the results might not be applicable to other populations. Finally, this was a retrospective study, and we found that in our study SEPC patients and OPEC patient had similar clinicopathological characteristics and prognosis and there was little difference in management between SEPC and other tumors, to confirm the results, large randomized controlled trial studies may be required. Nonetheless, the long follow-up and relatively larger sample size of our study provided us with better data than other studies, to evaluate the risk for SEPC, and to provide a novel, predictive model of SEPC prognosis.

\section{Conclusions}

Patients with SEPC appeared with unique clinical pathological features compared with OPEC patients, including older age, more squamous cell carcinoma, early stage esophageal cancer, and lower third of esophageal cancer, while SEPC patients and OPEC patients had similar survival curves. $M$ stage, surgery, and chemotherapy were independently associated with OS. A nomogram prediction based on $M$ stage, surgery, and chemotherapy was used to predict 1-, 3-, and 5-year OS of these patients.

\section{Acknowledgments}

We would like to thank International Science Editing Co. for editing the language.

Funding: This work was supported by the National Natural Science Foundation of China (Grant No. 81572295), and the Shanghai Sailing Program (No.17YF1402400).

\section{Footnote}

Conflicts of Interest: The authors have completed the ICMJE uniform disclosure form (available at http://dx.doi. org/10.21037/tcr.2019.12.64). The authors have no conflicts of interest to declare.

Etbical Statement: The authors are accountable for all aspects of the work in ensuring that questions related to the accuracy or integrity of any part of the work are appropriately investigated and resolved. Ethics approval was exempted by the Ethics Committee of the Zhongshan Hospital, Fudan University (Shanghai, China), as the SEER is a publicly available database, and data extracted from SEER were identified as an exempted study.

Open Access Statement: This is an Open Access article distributed in accordance with the Creative Commons Attribution-NonCommercial-NoDerivs 4.0 International License (CC BY-NC-ND 4.0), which permits the noncommercial replication and distribution of the article with the strict proviso that no changes or edits are made and the original work is properly cited (including links to both the formal publication through the relevant DOI and the license). See: https://creativecommons.org/licenses/by-nc-nd/4.0/.

\section{References}

1. Bray F, Ferlay J, Soerjomataram I, et al. Global cancer statistics 2018: GLOBOCAN estimates of incidence and mortality worldwide for 36 cancers in 185 countries. CA Cancer J Clin 2018;68:394-424.

2. Saeki H, Tsutsumi S, Yukaya T, et al. Clinicopathological Features of Cervical Esophageal Cancer: Retrospective Analysis of 63 Consecutive Patients Who Underwent Surgical Resection. Ann Surg 2017;265:130-6.

3. Xia H, Shen J, Chen S, et al. Overexpression of VEGF-C correlates with a poor prognosis in esophageal cancer patients. Cancer Biomark 2016;17:165-70.

4. Huang FL, Yu SJ. Esophageal cancer: Risk factors, genetic association, and treatment. Asian J Surg 2018;41:210-5.

5. Donin N, Filson C, Drakaki A, et al. Risk of second primary malignancies among cancer survivors in the United States, 1992 through 2008. Cancer 2016;122:3075-86.

6. Rice TW, Ishwaran H, Ferguson MK, et al. Cancer of the Esophagus and Esophagogastric Junction: An Eighth Edition Staging Primer. J Thorac Oncol 2017;12:36-42. 
7. Wang Y, Li J, Xia Y, et al. Prognostic nomogram for intrahepatic cholangiocarcinoma after partial hepatectomy. J Clin Oncol 2013;31:1188-95.

8. Baba Y, Ishimoto T, Kurashige J, et al. Epigenetic field cancerization in gastrointestinal cancers. Cancer Lett 2016;375:360-6.

9. Bugter O, Spaander MCW, Bruno MJ, et al. Optical detection of field cancerization in the buccal mucosa of patients with esophageal cancer. Clin Transl Gastroenterol 2018;9:152.

10. Slaughter DP, Southwick HW, Smejkal W. Field cancerization in oral stratified squamous epithelium; clinical implications of multicentric origin. Cancer 1953;6:963-8.

11. Muller J, Grosclaude P, Lapotre-Ledoux B, et al. Trends in the risk of second primary cancer among bladder cancer survivors: a population-based cohort of 10047 patients. BJU Int 2016;118:53-9.

12. Chang P, Regan MM, Ferrer M, et al. Relief of Urinary Symptom Burden after Primary Prostate Cancer Treatment. J Urol 2017;197:376-84.

13. Murray L, Henry A, Hoskin P, et al. Second primary cancers after radiation for prostate cancer: a systematic review of the clinical data and impact of treatment technique. Radiother Oncol 2014;110:213-28.

14. Katada C, Yokoyama T, Yano T, et al. Alcohol Consumption and Multiple Dysplastic Lesions Increase Risk of Squamous Cell Carcinoma in the Esophagus, Head, and Neck. Gastroenterology 2016;151:860-9.e7.

15. Utada M, Ohno Y, Hori M, et al. Incidence of multiple primary cancers and interval between first and second primary cancers. Cancer Sci 2014;105:890-6.

16. Shirai K, Tamaki Y, Kitamoto Y, et al. Prognosis was not deteriorated by multiple primary cancers in esophageal cancer patients treated by radiotherapy. J Radiat Res 2013;54:706-11.

17. Noronha V, Joshi A, Patil VM, et al. Efficacy and Safety of Induction Chemotherapy in Esophageal Cancer with Airway Involvement. J Gastrointest Cancer 2016;47:294-304.

18. Levi F, Randimbison L, Blanc-Moya R, et al. High constant incidence of second primary colorectal cancer. Int J Cancer 2013;132:1679-82.

19. Sud A, Thomsen H, Sundquist K, et al. Risk of Second Cancer in Hodgkin Lymphoma Survivors and Influence of Family History. J Clin Oncol 2017;35:1584-90.

20. Lee JS, Ahn JY, Choi KD, et al. Synchronous second primary cancers in patients with squamous esophageal cancer: clinical features and survival outcome. Korean J
Intern Med 2016;31:253-9.

21. Kudou M, Shiozaki A, Fujiwara H, et al. Clinical analysis of esophageal cancer patients with a history of metachronous primary cancer. Gan To Kagaku Ryoho 2014;41:2030-2.

22. Otowa Y, Nakamura T, Takiguchi G, et al. Safety and benefit of curative surgical resection for esophageal squamous cell cancer associated with multiple primary cancers. Eur J Surg Oncol 2016;42:407-11.

23. Poon RTP, Law SYK, Chu K-M, et al. Multiple Primary Cancers in Esophageal Squamous Cell Carcinoma: Incidence and Implications. The Annals of Thoracic Surgery 1998;65:1529-34.

24. Baba Y, Yoshida N, Kinoshita K, et al. Clinical and Prognostic Features of Patients With Esophageal Cancer and Multiple Primary Cancers: A Retrospective Singleinstitution Study. Ann Surg 2018;267:478-83.

25. Kumagai Y, Kawano T, Nakajima Y, et al. Multiple primary cancers associated with esophageal carcinoma. Surg Today 2001;31:872-6.

26. Natsugoe S, Matsumoto M, Okumura H, et al. Multiple primary carcinomas with esophageal squamous cell cancer: clinicopathologic outcome. World J Surg 2005;29:46-9.

27. Ariyoshi R, Toyonaga T, Tanaka S, et al. Clinical outcomes of endoscopic submucosal dissection for superficial esophageal neoplasms extending to the cervical esophagus. Endoscopy 2018;50:613-7.

28. Shi Q, Sun D, Cai SL, et al. Clinical Analysis of Endoscopic Submucosal Dissection for the Synchronous Multiple Primary Early Cancers in Esophagus and Stomach: 12 Cases Report. J Laparoendosc Adv Surg Tech A 2018;28:1068-73.

29. Lee GD, Kim YH, Kim JB, et al. Esophageal Cancer Associated with Multiple Primary Cancers: Surgical Approaches and Long-term Survival. Ann Surg Oncol 2013;20:4260-6.

30. Li QW, Zhu YJ, Zhang WW, et al. Chemoradiotherapy for Synchronous Multiple Primary Cancers with Esophageal Squamous Cell Carcinoma: a Case-control Study. J Cancer 2017;8:563-9.

Cite this article as: Chen Z, Li M, Ma K, Hu Z, Wang S, Chen H, Zheng Y, Zhan C, Lin Z, Wang Q. Clinicopathological features and prognosis of patients with esophageal cancer as the second primary cancer: a large population-based analysis using the SEER program [2000-2015]. Transl Cancer Res 2020;9(2):1113-1124. doi: 10.21037/tcr.2019.12.64 
Supplementary

Table S1 Sits of FPC

\begin{tabular}{lc}
\hline Site of FPC & Total (\%) \\
\hline Skin* & $36(4.4)$ \\
Lymphoma & $47(5.8)$ \\
Colon and rectum & $80(9.8)$ \\
Urinary system & $83(10.2)$ \\
Oral cavity and pharynx & $85(10.5)$ \\
Respiratory system & $100(12.3)$ \\
Genital system & $354(43.5)$ \\
Others & $28(3.5)$ \\
Total & $813(100)$ \\
\hline
\end{tabular}

*, skin excluding basal and squamous. FPC, first primary cancer prior to esophageal cancer.

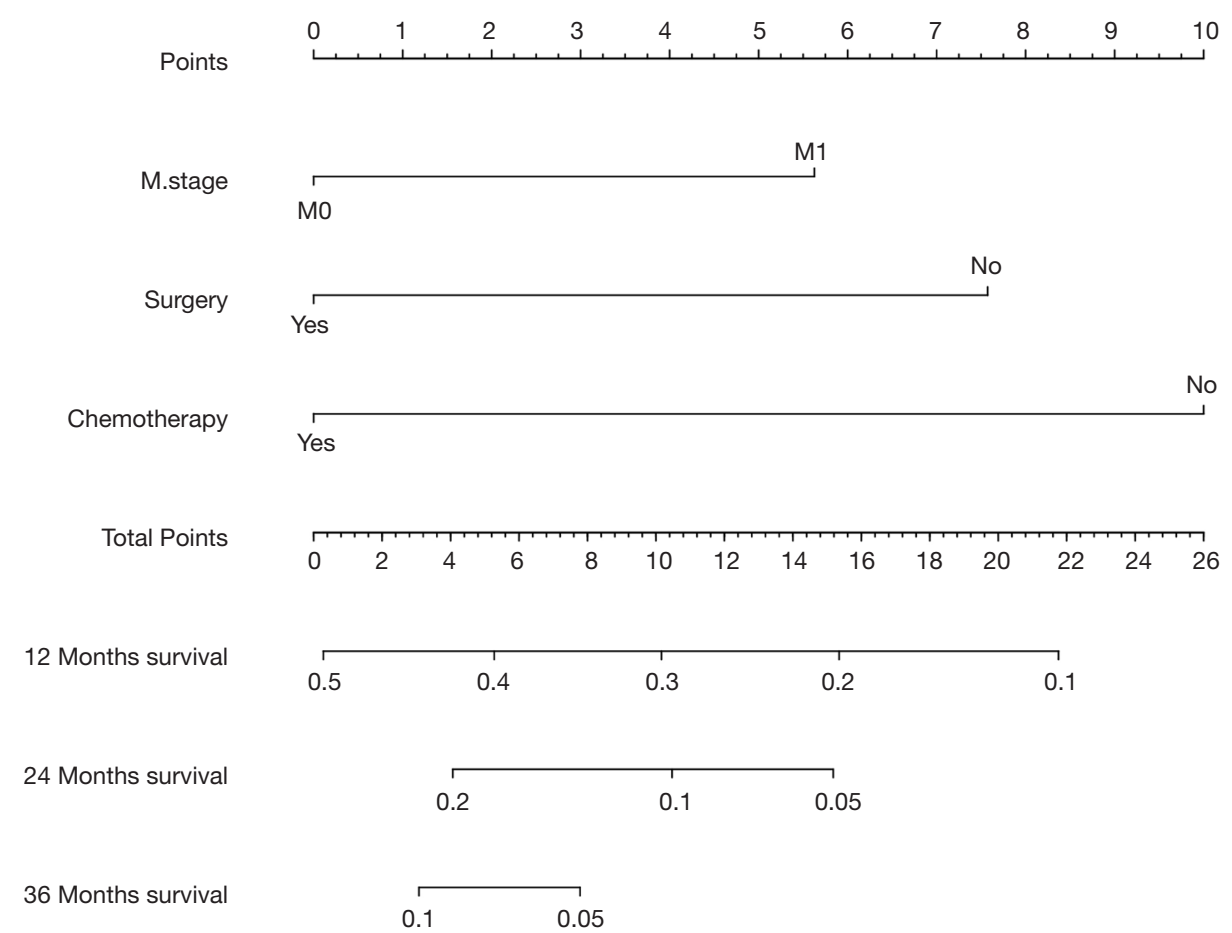

Figure S1 Nomogram to predict 1-, 2-, and 3-year overall survival of patients with SEPC. SEPC, second primary cancer. 


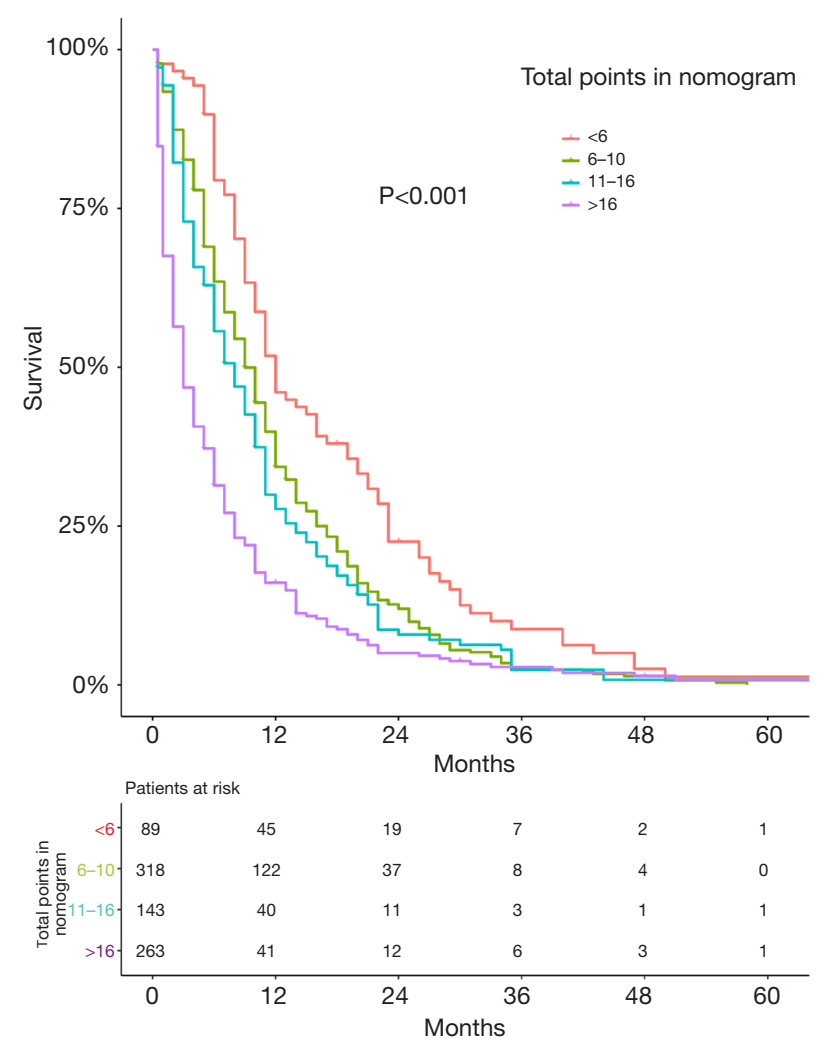

Figure S2 Kaplan-Meier estimate of overall survival in patients treated by total points in nomogram. 